\title{
Computer Aided Optical Disc Detection in Fundus Images: A Review
}

\author{
G.N. Balaji ${ }^{1}$, S V. Suryanarayana ${ }^{2}$, Naresh Babu Kakarla ${ }^{3}$ \\ 1, Asst. Professor, CVR College of Engineering/IT Department, Hyderabad, India \\ balaji.gnb@gmail.com \\ ${ }^{2}$ Assoc. Professor, CVR College of Engineering/IT Department, Hyderabad, India \\ suryahcu@gmail.com \\ ${ }^{3}$ Asst. Professor, CVR College of Engineering/CSE Department, Hyderabad, India \\ nareshbabukakarla@gmail.com
}

\begin{abstract}
Colour fundus images of the human retina are increasingly used in the diagnosis and treatment of several eye related pathologies and in ailments such as arteriosclerosis, diabetes, and hypertension. The effectiveness of treatment for many eye related diseases lies in the early detection through regular screenings. But, screening a large number of patients is a significant problem faced by medical practitioners in populous developing countries like India. In addition, there are extensive influences of human blunders and subjectivity on the consequences of assessment by a human master. This opens up the probability of applying propelled image processing techniques in fundus images to support and improve determination in different ways. Thus, automatic detection of pathologies and computer aided analysis in retinal images play a important role in modern diagnostic procedures and screening systems. Reliable and robust extraction of retinal features like optic disc, macula and vasculature is an essential for ensuing retinal image analysis and handling since these are the overwhelming and most stable structures showing up in the retina. However, automatic segmentation of retinal images is a complicated affair since retinal images are often poorly contrasted noisy, and there are a wide variations in orientation. This paper presents a review on algorithms or methods to detect optic disc automatically.
\end{abstract}

Index Terms: diabetes, hypertension, retina, optical disc and arteriosclerosis.

\section{INTRODUCTION}

The human eye is regularly contrasted with a camera. Approaching light reflected from objects is centered around to the retina in the track of passing through cornea, understudy and focal point, which is like the component of a camera. A camera utilizes the photographic film or a CCD board to make a picture, while the eye utilizes a specific layer of cells, called the retina, to create a image. The approaching data is prepared by photoreceptor cells in the retina and transmitted to the mind through optic nerve. The eye's astonishing capacity to concentrate on an extensive variety of articles having distinctive sizes, glow and difference at a rapid is more effective than those of presently accessible cameras.

A fundus camera is used to capture the images of the interior surface of the retina. With its digital imaging capabilities, the images may be enhanced, stored and retrieved more easily than film, and images may be transferred electronically to other sites for a trained optical technician or an expert ophthalmologist to detect or diagnose diseases for a patient at a distant location. In the fundus image of a healthy human subject, features such as optic disc, retinal vasculature and macula are visible as shown in Figure 1.

There are two sorts of value issues in the fundus images: noise pixels and pixels whose color is twisted. Both appear to exist in locales where light has been deficient. Since brightening is normally sufficient in the focal point of the picture, poor picture quality districts are situated close to the edge of the fundus.

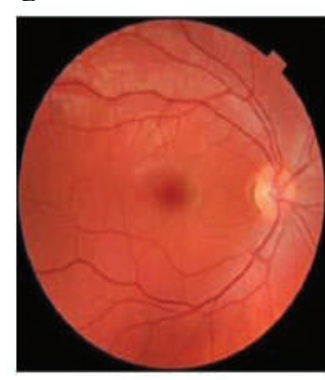

(a)

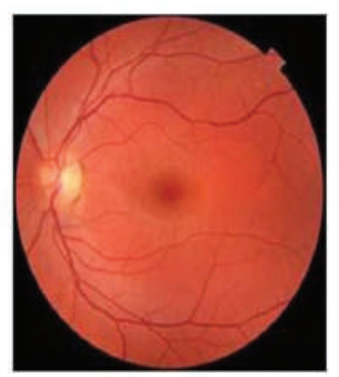

(b)
Figure 1. Fundus images of both eyes

Figure 2 demonstrates a solid retinal image with fundamental retinal highlights i.e. veins, macula and optic disk. The color size, shape of optic disk help in discovery and restriction, as it may demonstrate a vast difference.

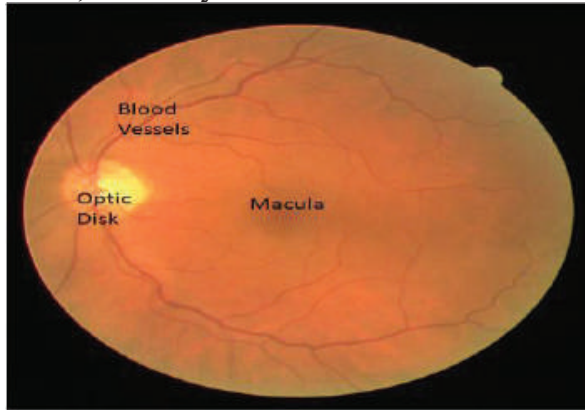

Figure 2. Healthy retinal image with features

Figure 3 indicates cases of a swollen optic nerve, where the circular shape and size are twisted. It likewise 
demonstrates a case with a bright circular lesion that seems like optic disk.
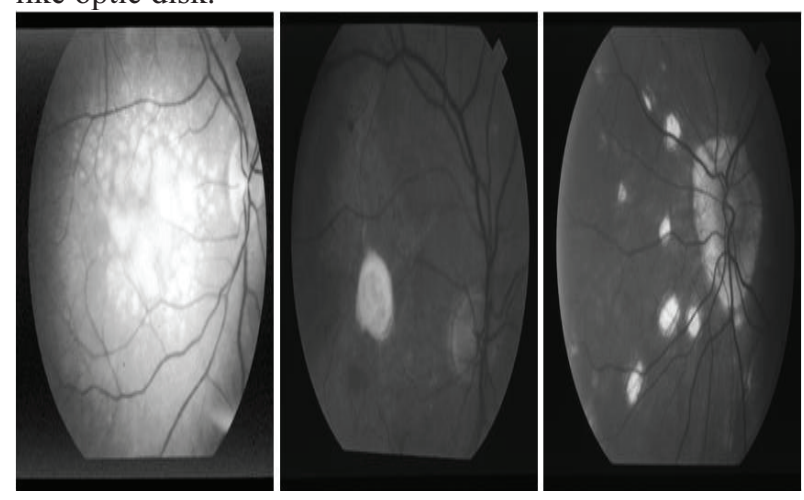

Figure 3. Retinal images with distorted shape of optic disk and lessions.

\section{A. Fundus image preprocessing}

Inappropriate scene illumination and in addition nonperfect procurement conditions due to misadjusted imaging framework can introduce serious distortions into the resulting image. These distortions are generally seen as smooth intensity varieties over the image. With such unevenness, the consequent image processing operations like image registration, segmentation, or pattern recognition might be confounded; in this way, the adjustment of illumination in homogeneities is exceedingly alluring. The objective of illumination adjustment is to evacuate uneven illumination of the image caused by sensor defaults, nonuniform illumination of the scene, or introduction of the surface. The known illumination revision techniques in the writing can be classified in the following gatherings: filtering segmentation based, surface fitting, and different strategies [1].

Particular techniques for illumination correction were proposed in the casing work of retinal picture handling and examination. Basic and quick techniques using large-kernel median filter to obtain low-pass correction coefficients were utilized by [2],[3] show the background of a fundus picture as a white Gaussian arbitrary field and utilize Mahalanobis distance for background pixel classification. Contrast normalization using high-pass filtering is utilized as a part of [4] as one stage of microaneurysm detection method. Added substance model of non-uniform illumination is utilized as a part of [5], together with adaptive histogram equalization. In [6-9], the nearby difference improvement strategy is utilized for adjusting uneven illumination in the intensity channel of fundus images. In [10] and [11], a substantial mean channel, vast middle channel, or both are utilized for evaluating the fundus foundation. Illumination variety in a fundus image can be dispensed by subtracting the foundation estimation from the first image or by partitioning the first image by the foundation estimation. Barely any different techniques are likewise displayed in the writing. For instance, in [12], the intensity esteems in dim areas have been expanded. Every illumination equalization strategy has its own points of interest and impediments, yet no method was discovered that would totally take care of the issue of uneven illumination. Illumination correction based on background subtraction is one of the widely accepted methods in fundus image preprocessing. Uneven illumination in a fundus image can be corrected by subtracting the background estimation from the original image or by dividing the original image by an estimated background.

\section{OPTIC DISC DETECTION}

Automatic detection and localization of optic disc is of paramount importance in all image processing applications with fundus images. Current optic disc detection methods available in the literature can be broadly classified in to intensity-based [7] [13-14], template-based [9] [15-21], shape-based [22-23] and vessel-based [5] [24-30] approaches. However most of the existing OD detection methods can be classified as given below.

\section{A. Methods based on Intensity}

A variance-based OD-detection method is given by [7]. This method assumes the appearance of the optic disc as a yellowish region typically occupying approximately one seventh of the entire image. The intensity variance of the image should be at its highest within the optic disc because of relatively rapid variation in intensity values. The reason for this variation is the appearance of dark blood vessels besides bright nerve fibers. The variance-based ODdetection method can be divided into three different steps:

- Local contrast enhancement

- Determination of the variance within a running window

- Determination of the average variance within a running window

The authors in [37] have tried optic disk localization and detection system on standard diabetic retinopathy databases. They have utilized four openly accessible datasets, DRIVE, diaretdb1, STARE and diaretdb0. The choice for fruitful localization or fizzled localization depends on human eye perception. Figure 4 represents the optic disk localization occurs for retinal images taken from various databases. Red cross sign demonstrates the optic disk area in each picture. These outcomes bolster the legitimacy of our procedure and demonstrate that our strategy gives great outcomes for localization notwithstanding for those images where it is hard to find optic disk. Similar kind of segmentation techniques are added with mathematical morphological operations and used for segmentation of irregular shapes from echocardiogram images in [39]. This method aided them to classify the basic cardiac views, also acts as an initial stage for computer aided cardiac disease diagnosis system. 


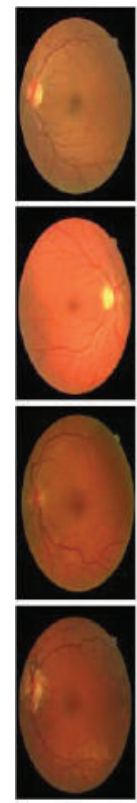

(a)
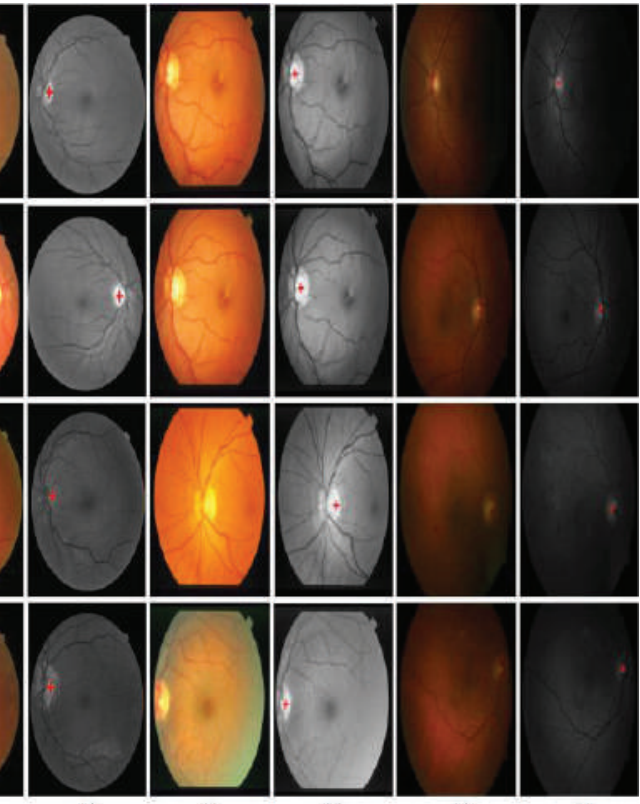

(b)
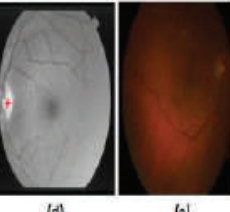

(d)

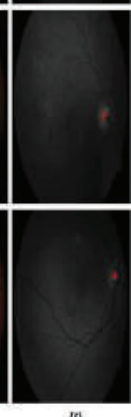

(f)

Figure 4. (a) DRIVE database; (b) OD localization in DRIVE (c) STARE database; (d) OD localization in STARE; (e) Diarectdb0 and Diarectdb1 database; (f) results of OD localization.

This method localizes the optic disc merely by means of its high grey level variation. However no method is suggested for the detection of the contours in this work. The techniques reported so far, based on luminosity, however fail on images with severe pathologies. It simply misses to locate the OD where the optic disc is obscured by hemorrhages or with a dark pigmentation. The present approach based on bit plane decomposition and mathematical morphology is an attempt to address the above problem.

\section{B. Methods based on Template Matching}

Template matching is a classical way to find the target object in an image [15-19], [31] employed Principal Component Analysis (PCA) to extract features of the optic disc. They first manually cut sub-images around the optic disc region from the training images and then used PCA to obtain the "eigen-discs" that describes the "disc-space". Using a template moving throughout a retinal image, the candidate sub-images were projected onto the disc-space and the candidate sub-image with the smallest reconstruction error was regarded as the optic disc. In their paper [9], [21] proposed to locate the optic disc by using a template matching approach based on a normalized correlation coefficient. The normalization of the colour fundus image is performed by applying histogram specification on each colour plane (R,G \& B). Then the optic disc region from 25 normalized images were averaged to produce a template. Finally they used the normalized correlation coefficient to find the most perfect match between the template and all the candidate sub-images.

The authors in the paper [29-30] mentioned that the approach can possibly recognize the area of the OD in retinal images with few or no variations from the norm and the position in the image which is situated amidst the optic circle. To achieve this they utilized $\mathrm{k}-\mathrm{NN}$ regression to discover the connection between the reliant variable $\mathrm{d}$, speaking to the separation to the optic plate focus, and a feature vector measured around a round layout. The format is round in light of the fact that the optic circle is roughly circular by itself. The round format is put at different areas in the picture and measures the features around the layout. In view of the feature vector the separation of circular layout is utilized and free factors $\mathrm{v}$ is measured at the outskirt and under the format. The training set is made putting away separation $d$ to genuine protest focus. The estimated OD Distance d, d can be calculated by:

$\widetilde{d}=\frac{1}{k} \sum_{i=1}^{k} d_{i}$

Where $i d$ is the stored value of $\mathrm{d}$ of the $\mathrm{i}^{\text {th }}$ nearest neighbor of $\mathrm{v}$ and $d$ location of object of interest that is get detected.

In [20], the authors Proposed a Hausdorff-based template matching. They estimate the OD contour using Hausdorffbased matching between detected edges and a circular template. In this the OD detection method is primarily based on pyramidal decomposition. First, a multi-resolution processing was employed through pyramidal decomposition. Small bright lesions were eliminated at lower resolutions, which speed up the searching for the optic disc since it reduces the number of false candidates. A confidence value was calculated for all the candidate regions, and then the Canny edge detector [32] was applied on the green channel image regions corresponding to the candidate regions to construct a binary edge map. Finally, the Hausdorff distance was used to match the edge map regions to a circular template of various different radii. It has been reported that this method is not a robust one particularly in images with multiple pathologies and hemorrhages in the vicinity of optic disc.

\section{Methods based on the Largest Object}

The authors in [14] detected the optic disc as the largest and brightest object in the retina by assuming that all bright lesions are much smaller than the size of the optic disc. In this method of OD-localization a threshold is applied. A straightforward edge is connected to get a twofold picture containing parts of the optic disc and other splendid showing up pathologies like exudates. The biggest associated question inside the thresholded image is relied upon to be a piece of the optic plate. The focal point of this problem is chosen as the focal point of the optic circle. Here, a territory edge is utilized to localize the optic plate and the watershed change to discover its forms. Shape anomalies because of segmentation mistakes, especially with regards to active vessels or in low complexity couldn't have been disposed of. Likewise [33] utilizes a comparative approach. In any case, the calculation comes up short when contrast is too low or when the red channel is soaked. 


\section{Methods based on Hough Transform}

In [22], the author proposes to apply a Hough transform in order to locate the optic disc the Hough transform system can discover geometric shapes in an image. Objects of geometric shapes might be identified by changing over the condition of the protest into a Hough space parameter condition. For instance, a line and a circle can be spoken to in Hough space by:

$$
\begin{aligned}
& \text { line: } x i \cos \theta+y i \sin \theta=\rho \\
& \text { circle: }(x i-a)^{2}+(y i-b)^{2}=c^{2}
\end{aligned}
$$

There are two parameters for line and three parameters for circle in Hough space, center $(a, b)$ and the range $c$ of the circle. The optic circle has a roughly round shape, along these lines the Hough transform can be utilized to recognize the optic plate. With the optic circle span settled in Hough parameter space, the search for a round protest turns into a two-dimensional issue.

This technique finds the round shape with settled span in a thresholded edge image of the fundus. To distinguish edges of every conceivable introduction at every pixel in an image compass edge recognition with a Sobel kernel is connected. The maximal reaction of the Sobel kernel for every introduction is held. On this edge guide of the retinal surface a solitary edge is connected to acquire a paired edge outline. Atlast the Hough transform strategy is connected to the edge pixels in the edge guide to aggregate proof of circles with settled range $\mathrm{c}$ in the image. The circle with the highest magnitude of evidence is chosen as the optic disc. The technique used in [34] is again a Hough transform based method to detect the contours of the optic disc. Obviously, some improvements have been made in this, but problems have been stated if the optic disc does not meet the shape conditions (e.g., if it lies on the border of the image) or if contrast is very low.

In [23] It was detected, that the optic disc using the circular Hough transform (CHT). First the retinal vessels were suppressed using a morphological closing operator. Then the Sobel operator was used to extract the edges in the image. Finally the CHT was applied to the edge map, and the optic disc was identified as the largest circle.

\section{E. Methods based on Supervised Learning}

In [24], [25] proposed a technique for optic nerve location in view of a calculation called fuzzy convergence. This calculation distinguished the optic nerve as the point of convergence of the vein arrange. Fuzzy convergence utilizes the endpoints of the vein segments to help discover the arrangement. For every partition $p$ it uses the following equation for discriminant statistics as follows:

$$
F_{P}=\frac{\left(\mu_{A}-\mu_{B}\right)^{2}}{\left(\sigma_{A}^{2}+\sigma_{B}^{2}\right)}
$$

Where $\mu$ and $\sigma$ is the mean and standard deviation of each set. The largest value of indicates the best partition. The method used the convergence of the blood vessel network; this method also takes lot of time to execute on single image.

\section{F. Methods based on Vessel model}

[24] localized OD by back-tracking the vessels to their origin. It is one of the good methods to localize OD but the system has to rely on the vascular tree segmentation which is time consuming. Grison et al. proposed a technique that depends on a model of the geometrical directional example of the retinal vascular framework. This framework verifiably holds the data on the OD position as the purpose of vessel convergence. Utilizing the vessel focus line focuses and the comparing vessel bearings gave by any vessel ID technique, the model parameters were distinguished by a methods for reducing complexity. These evaluated esteems give the directions of the OD focus. [32] detected OD by phaseportrait analysis. Assuming OD is near the focal point of convergence of retinal vessel. The vasculature network is then extracted by Gabor filter. The $\mathrm{ONH}$ is obtained via phase portrait analysis and intensity based condition.

\section{Miscellaneous Methods}

Few other schemes for OD detection by Frank ter Har [35] described in the literature. These methods are based on pyramidal decomposition of the vasculature and the green plane, the branch with the most vessels, path convergence with the Hough transform and vasculature fitting on a directional model. The authors observed that the method which performs best is the vasculature fitting on a directional model. This method is found to be better than all other evaluated methods in their own database but it fails in STARE database.

The vasculature fitting on a directional model is described by the author in [35] as follows. This OD-detection method uses the orientation of the vasculature, which can be extracted from a fundus image to locate the optic disk. The idea to use vessel orientations to locate the optic disc was first introduced by the authors in [36]. They used a mathematical model that described the expected vessel orientation for each point in an image with respect to the optic disc. Then the sum of squared differences was used to compare the orientations of a new vasculature with the mathematical model, resulting in the OD-location. Starting at the optic disc, the vessels follow more or less the same divergence pattern in all retinal images. Four or five main vessels move in a vertical direction out of the optic nerve. Two of these main vessels curve away towards the macula and make sure that that part of th1111111111111e retina is supplied with blood, while the other main vessels diverge 
toward other parts of the retina. Branches of the main vessels are spread around the entire retina.

Branches coming out of the two main vessels near the macula converge toward the macula. The automatic ODdetection method here captures these properties in a directional model. Some of the algorithms discussed above will locate only the centre of optic disc, while another category of algorithms will locate it and encircle the area corresponding to optic disc. Very few algorithms are available in the literature for both localization and extraction of the optic disc pixels. The algorithms used today for optic disc localization and extraction are either computationally intensive or less accurate, particularly in the presence of anomalies like exudates in the human retina. Moreover the methods currently available for OD detection largely depends on a prior knowledge of other retinal features like vasculature for the accurate detection of OD. The authors in [37] presents an OD detection and segmentation methodology which is able to detect the OD center without using any template or prior vascular information. Table 1 shows comparative success rate results of existing methods with different databases.

TABLE I.

Comparative Success Rate Results Of Existing Methods With DIFFERENT DATABASES

\begin{tabular}{|c|c|c|c|c|}
\hline Sr No & $\begin{array}{l}\text { OD Detection } \\
\text { Methods }\end{array}$ & $\begin{array}{c}\text { Local } \\
\text { Database }\end{array}$ & $\begin{array}{l}\text { STARE } \\
\text { Database }\end{array}$ & $\begin{array}{c}\text { Drive } \\
\text { Database }\end{array}$ \\
\hline 1. & $\begin{array}{l}\text { Template Matching } \\
\text { and Matched Filter }\end{array}$ & $100 \%$ & $98.8 \%$ & - \\
\hline 2. & $\begin{array}{l}\text { Circular Template } \\
\text { Matching and KNN } \\
\text { regression }\end{array}$ & $99.9 \%$ & - & - \\
\hline 3. & $\begin{array}{l}\text { Hausdorff based } \\
\text { Template Matching }\end{array}$ & $100 \%$ & 71.6 & - \\
\hline 4. & PCA based Method & $99 \%$ & - & - \\
\hline 5. & Haar Based DWT & 89 & 70.4 & - \\
\hline 6. & $\begin{array}{l}\text { Pyramidal } \\
\text { Decomposition }\end{array}$ & 93.2 & 54.3 & - \\
\hline 7. & Fuzzy Convergence & 97.4 & 65.4 & - \\
\hline 8. & $\begin{array}{l}\text { Pyramidal } \\
\text { decomposition of both } \\
\text { vasculature and green } \\
\text { plane }\end{array}$ & 93.2 & 54.3 & - \\
\hline 8. & $\begin{array}{l}\text { Gabor filter and Face } \\
\text { portrait analysis }\end{array}$ & - & 69 & 100 \\
\hline 9. & Morphology Based & 100 & 58 & - \\
\hline 10. & Variation of Intensity & 99.1 & 42 & - \\
\hline 12. & $\begin{array}{l}\text { Circular Hough } \\
\text { transform and grow- } \\
\text { cut algorithm } \\
\text { Abdullah et al. }[37]\end{array}$ & 100 & 98.68 & 100 \\
\hline
\end{tabular}

(-) indicates information is provided in relevant field.

Images of the ocular fundus can convey the retinal, ophthalmic, and even systemic diseases such as diabetes, hypertension, and arteriosclerosis. The use of colour retinal images captured with digital fundus cameras provides a noninvasive way of search and screening for ophthalmic and systemic pathologies. A completely automated segmentation of colour retinal images can greatly help in the management of certain diseases like diabetic retinopathy which require the screening of large populations. But the development of an automated system for this purpose needs a robust system capable of fast segmentation of the normal anatomical features of the retina which is not yet available.

\section{CONCLUSIONS}

Unfortunately a large portion of the calculations utilized today for macula localization or fovea detection are computationally concentrated, requires earlier data on OD, and are less exact, especially within the sight of pathologies in the human retina. These downsides can be tended to, and a novel approach based OD can be considered for future work. This will permit to better recognize the framework insufficiencies and to propose arrangements, conceivably by joining existing methodologies.

The most challenging part of OD segmentation is preprocessing as each fundus image taken vary by contrast, color etc. The existing algorithms does not work well for the entire standard databases which are publically available. Circular Hough transform and grow-cut algorithm (GC) is computationally fast in processing, robust to the variation in image contrast and illumination, works well in pathological retinal images and is comparable with state-of-the-art methodologies in terms of quantitative performance metrics. Thus, OD segmentation in medical imaging is the challenging job for the researchers preprocessing the original image before further processing on it is key step in detection of OD. Better preprocessing gives the OD segmentation results up to the mark. Hence there is a need of OD detection algorithms which gives the better performance on noisy databases.

\section{REFERENCES}

[1] Kubecka, Libor., Jan, Jiri. and Kolar, Radim. Retrospective Illumination Correction of Retinal Images, International Journal of Biomedical Imaging, 2010.

[2] Niemann, H., Chrastek, R. and Lausen , B. "Towards automated diagnostic evaluation of retina images," Pattern Recognition and Image Analysis, Vol. 16, No. 4, pp. 671-676, 2006

[3] Foracchia, M. , Grisan, E. and Ruggeri, A. "Luminosity and contrast normalization in retinal images," Medical Image Analysis, Vol. 9, No. 3, pp. 179-190, 2005.

[4] Fleming, A. D., Philip, S., Goatman, K. A., Olson, J. A. and Sharp, P. F. "Automated microaneurysm detection using local contrast normalization and local vessel detection," IEEE Transactions on Medical Imaging, Vol. 25, No. 9, pp. 1223 1232, 2006.

[5] Abdel-Razik Youssif, A.A.H. , Ghalwash, A. Z. and AbdelRahman Ghoneim, A. A. S. "Optic disc detection from normalized digital fundus images by means of a vessels' direction matched filter," IEEE Transactions on Medical Imaging, Vol. 27, No. 1, pp. 11-18, 2008.

[6] Usher, D., Dumskyj, M., Himaga, M. , Williamson, T. H. , Nussey, S. and Boyce, J., "Automated detection of diabetic retinopathy in digital retinal images: a tool for diabetic 
retinopathy screening", Diabetes UK, Diabetic Medicine, Vol. 21, pp.84-90, 2003

[7] Sinthanayothin,C, Boyce, J. F., Cook, H. L. and Williams, T. $\mathrm{H}$. "Automated localisation of the optic disc, fovea and retinal blood vessels from digital colour fundus images", British J. of Ophthalmology, Vol. 83, No.8, pp. 902-910, Aug 1999

[8] Sinthanayothin,C.,Kongbunkiat, V., Phoojaruenchanachain, S. and Singlavanija, A. "Automated screening system for diabetic retinopathy", Proceedings of the 3rd International Symposium on Image and Signal Processing and Analysis, pp. 915-920, 2003.

[9] Osareh, A. "Automated identification of diabetic retinal exudates and the optic disc," Ph.D. dissertation, Department of Computer Science, Faculty of Engineering, University of Bristol, Bristol, U.K., 2004.

[10] Phillips ,Russell, John, Forrester. and Peter "Sharp. Automated detection and quantification of retinal exudates. Graefe's Archive for Clinical and Experimental Ophthalmology", Vol.231, pp.90-94, 1993.

[11] Bernhard M. Ege, Ole K. Hejlesen, Ole V. Larsen, Karina Moller,Barry Jennings, David Kerr, and David A. Cavan, "Screening for diabetic retinopathy using computer based image analysis and statistical classification", Computer Methods and Programs in Biomedicine, Vol.62, pp.165-175, 2000.

[12] Wang, Huan., Hsu, Wynne., Kheng Guan Goh and Mong Li Lee, "An effective approach to detect lesions in color retinal images", IEEE Conference on Computer Vision and Pattern Recognition (CVPR), South Carolina, USA, pp. 181-187, June 2000

[13] Sinthanayothin, C. , Boyce, J. F. , Williamson, T. H. , Cook, H. L. , Mensah, E. , Lal, S. and Usher, D. "Automated detection of diabetic retinopathy on digital fundus images", Diabet. Med., Vol. 19, pp. 105-112, 2002.

[14] Walter, T. and Klein, J.C. "Segmentation of color fundus images of the human retina: Detection of the optic disc and the vascular tree using morphological techniques", Proceedings of the 2nd International Symposium on Medical Data Analysis, pp. 282-287, 2001.

[15] Li, H. and Chutatape, O. "Automated Feature Extraction in Color Retinal Images by a Model Based Approach", IEEE Trans. on Medical Imaging, Vol. 51, No.2, pp. 246-254,2004.

[16] Li, H. and Chutatape, O. "A model-based approach for automated feature extraction in fundus images," 9th IEEE Int. Conf. Computer Vision (ICCV'03), Vol. 1, pp. 394-399, 2003.

[17] Li, H. and Chutatape, O. "Boundary detection of optic disk by a modified ASM method", Pattern Recognition 36, pp. 20932104, 2003.

[18] Li, H. and Chutatape, O. "Automatic location of optic disc in retinal images," IEEE Int. Conf. Image Process., Vol. 2, pp. 837-840, 2001.

[19] Li, H. and Chutatape, O. "Fundus image features extraction", Proceedings of the 22th annual EMBS International conference,pp. 3071-3073, July 2000.

[20] Lalonde, M., Beaulieu, M. and Gagnon, L. "Fast and robust optic disc detection using pyramidal decomposition and Hausdorff-based template matching", IEEE Transactions on Medical Imaging, Vol.20, No.11, pp.1193-1200, 2001.

[21] Osareh, A., Mirmehdi, M., Thomas, B. and Markham, R. "Classification and localisation of diabetic-related eye disease,"7th Euro. Conf. Computer Vision (ECCV), LNCS, Vol. 2353, pp. 502-516, May 2002.

[22] Barrett, S.F., Naess, E. and Molvik, T. "Employing the Hough transform to locate the optic disk", Biomedical Sciences instrumentation, Vol. 37, pp.81-86, 2001.
[23] Abdel-Ghafar, R., Morris, T., Ritchings, T. and Wood, I. "Detection and characterisation of the optic disc in glaucoma and diabetic retinopathy," Med.Image Understand. Annual. Conf., London, U.K., Sep. 23-24, 2004.

[24] Hoover, A. and Goldbaum, M. "Fuzzy convergence," in Proc. IEEE Computer Soc. Conf. Computer Vis. Pattern Recognit., Santa Barbara, CA, pp. 716-721,1998.

[25] Hoover, A. and Goldbaum, M. "Locating the optic nerve in a retinal image using the fuzzy convergence of the blood vessels" IEEE Transactions on Medical Imaging, Vol.22, No.8, pp.951-958, 2003.

[26] Foracchia, M., Grisan, E. and Ruggeri, A. "Detection of optic disc in retinal images by means of a geometrical model of vessel structure," IEEE Transactions Medical Imaging, Vol. 23, No. 10, pp. 1189-1195, Oct. 2004.

[27] Lowell, J., Hunter, A. , Steel, D. , Basu, A., Ryder, R. , Fletcher, E. and Kennedy, L. "Optic nerve head segmentation," IEEE Transactions on Medical Imaging, Vol. 23, No. 2, pp. 256-264, Feb. 2004

[28] Tobin, K. W. , Chaum, E., Govindasamy, V. P. , Karnowski, T. P. and O. Sezer, "Characterization of the optic disc in retinal imagery using a probabilistic approach," Med. Imag. 2006: Image Process, Vol. 6144, pp. 1088-1097, 2006.

[29] Abràmoff, M. D. and Niemeijer, M. "The automatic detection of the optic disc location in retinal images using optic disc location regression," Proc. IEEE EMBC, pp. 4432-4435, Aug. 2006.

[30] Niemeijer, M. Abràmoff,M.D. "Segmentation of the Optic Disc, Macula and Vascular Arch in Fundus Photographs," IEEE Transactions on Medical Imaging, Vol. 26, No. 1, January 2007.

[31] Gonzales, R. C. , Woods R. E. and Eddins, S.L Digital Image Processing using MATLAB, Reading, Pearson Education Publishing Co., 2002

[32] Canny, J. "A computational approach to edge detection," IEEE Transactions on Pattern Recognition and Machine Intelligence, vol. PAMI-8, pp. 679-698, Nov. 1986.

[33] Walter, T., Klein, J. C. , Massin, P. and Erginay, A. "Detection of Exudates in Color Fundus Images of the Human Retina” IEEE Trans. Med. Imag., Vol. 21, No. 10, pp 12361243, October 2002.

[34] Pinz, A., BernÄogger, S., Datlinger, P. and Kruger, A. "Mapping the human retina", IEEE Transactions on Medical Imaging", Vol. 4, pp. 606-619, 2001.

[35] Frank ter Har, "Automatic Localization of Optic Disc in Digital Colour Images of the Human Retina", MS Thesis, Utrecht university, pp. 33-50, 2005

[36] Ruggeri, A., Foracchia, M. and Grisan, E. "Detection of optic disc based on a directional model of vascular network", http://www.cafia.org/cafia2003/cafia-3 abstracts.pdf.

[37] Abdullah et al. (2016), Localization and segmentation of optic disc in retinal images using circular Hough transform and grow-cut algorithm. PeerJ 4:e2003; DOI 10.7717/peerj.2003.

[38] Akram, Usman M., and Shoab A. Khan. "Automated detection of dark and bright lesions in retinal images for early detection of diabetic retinopathy." Journal of medical systems 36.5 (2012): 3151-3162.

[39] Balaji, G. N., T. S. Subashini, and N. Chidambaram. "Cardiac view classification using speed up robust features." Indian Journal of Science and Technology 8 (2015): 1. 